\title{
Orbifold Hodge Numbers of Calabi-Yau Hypersurfaces
}

\author{
Mainak Poddar \\ University of Wisconsin-Madison \\ Email: poddar@math.wisc.edu
}

\begin{abstract}
We identify the twisted sectors of a compact simplicial toric variety. We do the same for a generic nondegenerate Calabi-Yau hypersurface of an $n$-dimensional simplicial Fano toric variety and then explicitly compute $h_{\text {orb }}^{1,1}$ and $h_{\text {orb }}^{n-2,1}$ for the hypersurface. We give applications to the orbifold string theory conjecture and orbifold mirror symmetry.
\end{abstract}

\section{Contents}

\begin{tabular}{lll}
\hline 1 & Introduction & 2
\end{tabular}

$\begin{array}{lll}2 & \text { Orbifolds } & 3\end{array}$

2.1 Orbifold Structure . . . . . . . . . . . . . . . . . . . . 3

2.2 Orbifold Cohomology . . . . . . . . . . . . . . . . . 5

$\begin{array}{lll}3 & \text { Facts from Toric Geometry } & 7\end{array}$

3.1 Fan, Orbits and Divisors . . . . . . . . . . . . . . . . 7

3.2 Projective Toric variety from polytope . . . . . . . . . . . . . 9

3.3 Homogeneous coordinate ring . . . . . . . . . . . . . . . . . 10

3.4 Fano toric Varieties . . . . . . . . . . . . . . . . . . . . . . . 11

$\begin{array}{llr}4 & \text { Twisted Sectors } & 12\end{array}$

4.1 Twisted Sectors in Simplicial Toric Variety . . . . . . . . . . . 12

4.2 Twisted sectors of hypersurface of a Fano variety . . . . . . . 14 
$\begin{array}{lll}5 \text { Orbifold Hodge Numbers } & \mathbf{1 5}\end{array}$

$5.1 h_{a r b}^{1,1}(V) \ldots \ldots \ldots \ldots \ldots \ldots \ldots$

$5.2 h^{n-2,1}(V)$. . . . . . . . . . . . . . . . . . 16

5.3 Cohomology of the twisted sectors of $V$. . . . . . . . . . 20

5.4 Main Results . . . . . . . . . . . . . . . . . . . 22

5.5 An Example . . . . . . . . . . . . . . . . . . . . . . . . . . . 23

\section{Introduction}

Recently, there is an emerging subject of mathematics: Stringy geometry and topology of orbifolds. The core of this includes the developing theories of orbifold cohomology, orbifold quantum cohomology, orbifold K-theory, motivic integration, Mckay correspondence etc.

Physicists believe that orbifold string theory is equivalent to ordinary string theory of certain desingularisations. This belief motivated a body of conjectures, collectively referred to as the Orbifold string theory conjecture. The conjecture we are interested in is the K-Orbifold string theory conjecture. It states that there is a natural isomorphism between the Orbifold K-theory of a Gorenstein orbifold and the ordinary K-theory of its crepant resolution.

To construct a natural isomorphism as the conjecture demands, is a very hard problem. But many weaker versions of the conjecture that compare Euler numbers, Hodge numbers, etc. has been studied extensively in the literature in the case of orbifolds that are global-quotients. Batyrev[2], and Batyrev and Dais[4] proved, in particular, the equality of orbifold Hodge numbers and Hodge numbers of smooth crepant resolutions for Gorenstein global-quotient orbifolds. But there were no results for non global-quotient orbifolds.

In this paper, we show that the orbifold Hodge numbers of a generic CalabiYau hypersurface in a complex 4-dimensional simplicial Fano toric variety coincide with the Hodge numbers of its crepant resolution. Besides being the first non global-quotient example, this is also an important example in mirror symmetry. An immediate corollary of this is the mirror pairing of orbifold Hodge numbers for mirror families of Calabi-Yau 3-folds in simplicial Fano toric varieties. It should be remarked here that the orbifold structure on the Calabi-Yau hypersurface that we consider, is the one that naturally arises 
from its algebraic structure.

Recently the author has observed that one can combine the characterisation of twisted sectors in this paper with the apparatus of string-theoretic Hodge numbers developed by Batyrev and Dais [4], and Batyrev [2] to greatly generalise the results of this paper in the toric world. There is even a hope that the problem may be completely solved, as far as Hodge numbers go, by using motivic integration [7] and some stack theory. But those results will be discussed elsewhere.

Now we briefly describe how this article is organised. In sections 2 and 3 we review some facts from orbifold cohomology and toric geometry respectively. In section 4 we find characterisations for the twisted sectors of simplicial toric varieties and nondegenerate Calabi-Yau hypersurfaces of simplicial Fano toric varieties. In section 5 we compute formulas for some orbifold Hodge numbers of these hypersurfaces, state some corollaries and then give an example.

Acknowledgements: This work was done under the supervision of my doctoral advisor, Prof Yongbin Ruan. I am indebted to him for his guidance.

My special thanks are also due to Professors Igor Dolgachev, Lev Borisov and David Cox who were kind enough to listen to me and answer my questions. I thank Prof Cox for correcting an earlier draft of this article. I thank the faculty and friends at UW-Madison for their help and kindness.

\section{Orbifolds}

\subsection{Orbifold Structure}

Let $U$ be a connected topological space, $V$ a connected $n$-dimensional smooth manifold and $G$ a finite group acting smoothly on $V$. An $n$-dimensional uniformising system of $U$ is a triple $(V, G, \pi)$, where $\pi: V \rightarrow U$ is a continuous map inducing a homeomorphism between the quotient space $V / G$ and $U$. Two uniformising systems $\left(V_{i}, G_{i}, \pi_{i}\right), i=1,2$, are isomorphic if there is a diffeomorphism $\phi: V_{1} \rightarrow V_{2}$ and an isomorphism $\lambda: G_{1} \rightarrow G_{2}$ such that $\phi$ is $\lambda$-equivariant, and $\pi_{2} \circ \phi=\pi_{1}$.

Note: If $(\phi, \lambda)$ is an automorphism of $(V, G, \pi)$, then there is a $g \in G$ 
such that $\phi(x)=g . x$ and $\lambda(a)=g . a . g^{-1}$, for any $x \in V$ and $a \in G$.

Let $i: U^{\prime} \rightarrow U$ be a connected open subset of $U$. An uniformising system $\left(V^{\prime}, G^{\prime}, \pi^{\prime}\right)$ of $U^{\prime}$ is said to be induced from $(V, G, \pi)$ if there is a monomorphism $\lambda: G^{\prime} \rightarrow G$ and a $\lambda$-equivariant open embedding $\phi: V^{\prime} \rightarrow V$ such that $i \circ \pi^{\prime}=\pi \circ \phi$. The pair $(\phi, \lambda):\left(V^{\prime}, G^{\prime}, \pi^{\prime}\right) \rightarrow(V, G, \pi)$ is called an injection. Two injections $\left(\phi_{i}, \lambda_{i}\right):\left(V_{i}^{\prime}, G_{i}^{\prime}, \pi_{i}^{\prime}\right) \rightarrow(V, G, \pi), i=1,2$, are isomorphic if there is an isomorphism $(\psi, \tau)$ from $\left(V_{1}^{\prime}, G_{1}^{\prime}, \pi_{1}^{\prime}\right)$ to $\left(V_{2}^{\prime}, G_{2}^{\prime}, \pi_{2}^{\prime}\right)$, and an automorphism $(\bar{\psi}, \bar{\tau})$ of $(V, G, \pi)$ such that $(\bar{\psi}, \bar{\tau}) \circ\left(\phi_{1}, \lambda_{1}\right)=\left(\phi_{2}, \lambda_{2}\right) \circ(\psi, \tau)$.

Fact: Let $(V, G, \pi)$ be an uniformising system of $U$. For any connected open subset $U^{\prime}$ of $U,(V, G, \pi)$ induces a unique isomorphism class of uniformising systems of $U^{\prime}$.

Let $U$ be a connected and locally connected topological space. For any point $p \in U$ we define the germ of uniformising systems at $p$ in the following sense. Let $\left(V_{1}, G_{1}, \pi_{1}\right)$ and $\left(V_{2}, G_{2}, \pi_{2}\right)$ be uniformizing systems of neighbourhoods $U_{1}$ and $U_{2}$ of $p$. We say that $\left(V_{1}, G_{1}, \pi_{1}\right)$ and $\left(V_{2}, G_{2}, \pi_{2}\right)$ are equivalent at $p$ if they induce isomorphic uniformising systems for a neighbourhood $U_{3}$ of $p$.

Definition: Let $X$ be a Hausdorff, second countable topological space. An $n$-dimensional orbifold structure on $X$ is given by the following data : for any point $p \in X$, there is a neighbourhood $U_{p}$ and an $n$-dimensional uniformising system $\left(V_{p}, G_{p}, \pi_{p}\right)$ of $U_{p}$ such that for any point $q \in U_{p},\left(V_{p}, G_{p}, \pi_{p}\right)$ and $\left(V_{q}, G_{q}, \pi_{q}\right)$ are equivalent at $q$.

Definition: The germ of an orbifold structure on $X$ is an equivalence class under the following equivalence relation : two orbifold structures $\left\{\left(V_{p}, G_{p}, \pi_{p}\right)\right.$ : $p \in X\}$ and $\left\{\left(V_{p}^{\prime}, G_{p}^{\prime}, \pi_{p}^{\prime}\right): p \in X\right\}$ are equivalent if for any $p \in X,\left(V_{p}, G_{p}, \pi_{p}\right)$ and $\left(V_{p}^{\prime}, G_{p}^{\prime}, \pi_{p}^{\prime}\right)$ are equivalent at $p$. With a given germ of orbifold structures on it, $X$ is called an orbifold.

We call each $U_{p}$ a uniformised neighbourhood of $p$, and $\left(V_{p}, G_{p}, \pi_{p}\right)$ a chart at $p$. In fact we choose $U_{p}$ to be small enough that $G_{p}$ has the minimum possible order; that is, every element of $G_{p}$ fixes the preimage of $p$ in $V_{p}$. In what follows, this choice is assumed. 
An open subset $U$ of $X$ is called an uniformised open set if it is uniformised by $(V, G, \pi)$ such that for each $p \in U,(V, G, \pi)$ defines the same germ as $\left(V_{p}, G_{p}, \pi_{p}\right)$ at $p$. $X$ is called a global-quotient orbifold if $X$ itself is an uniformised open set. A point $p$ is called smooth or regular if $G_{p}$ is trivial; otherwise, it is called singular. The set of smooth points is denoted by $X_{\text {reg }}$, and the set of singular points is denoted by $X_{*}$.

An orbifold $X$ is called reduced if $G_{p}$ acts effectively on $V_{p}$. We can canonically associate a reduced orbifold to an orbifold by redefining the group actions. Furthermore if a group element acts nontrivially, we require that the fixed-point set is of at least (real) codimension two, so that the complement is locally connected. The toric varieties and hypersurfaces that we will deal with in this article are reduced orbifolds.

Remark: It is important to note here that even a reduced non-smooth orbifold can have a smooth underlying variety because of examples with complex reflections. For instance, consider $\mathbb{C} / \mathbb{Z}_{2}$ where $\mathbb{Z}_{2}$ acts by reflection about the origin. Then $\mathbb{C} / \mathbb{Z}_{2}$ is complex analytically isomorphic to $\mathbb{C}$ but it has a singularity at the origin in the orbifold sense. Gorenstein orbifolds do not present this problem as they do not admit such complex reflections.

\subsection{Orbifold Cohomology}

First we will describe the so-called twisted sectors. Consider the set of pairs:

$$
\tilde{X}_{*}=\left\{\left(p,(g)_{G_{p}}\right) \mid p \in X_{*}, 1 \neq g \in G_{p}\right\}
$$

where $(g)_{G_{p}}$ denotes the conjugacy class of $g$ in $G_{p}$. Then Kawasaki showed (see [5]) that $\tilde{X}_{*}$ is naturally an orbifold with an orbifold structure given by

$$
\left\{\pi_{p, g}:\left(V_{p}^{g}, C(g) / K_{g}\right) \rightarrow V_{p}^{g} / C(g) \mid p \in X_{*}, 1 \neq g \in G_{p}\right\}
$$

where $V_{p}^{g}$ is the fixed-point set of $g$ in $V_{p}, C(g)$ is the centralizer of $g$ in $G_{p}$, and $K_{g}$ is the kernel of the action of $C(g)$ on $V_{p}^{g}$. There is a map $\pi: \tilde{X}_{*} \rightarrow X$ defined by $\left(p,(g)_{G_{P}}\right) \mapsto p$ such that $\pi\left(\tilde{X}_{*}\right)=X_{*}$. In a sense $\tilde{X}_{*}$ is a resolution of singularities of $X$. 
Let $\tilde{X}=\tilde{X}_{*} \bigsqcup X$. We will describe the connected components of $\tilde{X}$. Recall that each point $p$ has a local chart $\left(V_{p}, G_{p}, \pi_{p}\right)$ which gives a local uniformized neighbourhood $U_{p}=\pi_{p}\left(V_{p}\right)$. If $q \in U_{p}$, up to conjugation, there is an injective homomorphism $G_{q} \rightarrow G_{p}$. For $g \in G_{q}$, the conjugacy class $(g)_{G_{p}}$ is well-defined. We define an equivalence relation $(g)_{G_{q}} \cong(g)_{G_{p}}$. Let $T$ denote the set of equivalence classes. By an abuse of notation, we use $(g)$ to denote the equivalence class to which $(g)_{G_{q}}$ belongs. $\tilde{X}$ is decomposed as a disjoint union of connected components

$\tilde{X}=\bigsqcup_{(g) \in T} X_{(g)}$,

where

$X_{(g)}=\left\{\left(p,\left(g^{\prime}\right)_{G_{p}}\right) \mid g^{\prime} \in G_{p},\left(g^{\prime}\right)_{G_{p}} \in(g)\right\}$.

Definition: $X_{(g)}$ for $g \neq 1$ is called a twisted sector. We call $X_{(1)}=X$ the nontwisted sector.

Let $T X$ denote the orbifold tangent bundle of $X$. See Chen and Ruan[5] for a definition. An almost complex structure $J$ on $X$ is a smooth section of the orbifold bundle $\operatorname{End}(T X)$ such that $J^{2}=-I d$. Assume that $X$ is an almost complex orbifold with an almost complex structure $J$. Then for any $p \in X_{*}, J$ gives rise to an effective representation $\rho_{p}: G_{p} \rightarrow G L(n, \mathbb{C})$. For any $g \in G_{p}$ we write $\rho_{p}(g)$, upto conjugation, as a diagonal matrix

$\operatorname{diag}\left(e^{2 \pi i \frac{m_{1, g}}{m_{g}}}, \ldots ., e^{2 \pi i \frac{m_{n, g}}{m_{g}}}\right)$, where $m_{g}$ is the order of $g$ in $G_{p}$, and $0 \leq m_{i, g}<$ $m_{g}$. Define a function $\iota: \tilde{X} \rightarrow \mathbb{Q}$ by

$$
\iota\left(p,(g)_{G_{p}}\right)=\sum_{i=1}^{n} \frac{m_{i, g}}{m_{g}} .
$$

This function $\iota: \tilde{X} \rightarrow \mathbb{Q}$ is locally constant. Denote it by $\iota_{(g)}$. It has the following properties:

(1) $\iota_{(g)}$ is integral iff $\rho_{p}(g) \in S L(n, \mathbb{C})$.

(2) $\iota_{(g)}+\iota_{\left(g^{-1}\right)}=\operatorname{rank}\left(\rho_{p}(g)-I d\right)=n-\operatorname{dim}_{\mathbb{C}} X_{(g)}$.

Definition: $\iota_{(g)}$ is called the degree shifting number of $X_{(g)}$.

Definition: An almost complex orbifold is called Gorenstein if property $(1)$ holds for all $(g)$.

Remark: An almost complex, complex or kahler structure on $X$ induces a corresponding similar structure on each $X_{(g)}$. 
Definition: Let $\mathbb{F}$ be any field containing $\mathbb{Q}$ as a subfield. We define the orbifold chomology groups of $X$ with coefficients in $\mathbb{F}$ by

$$
H_{\text {orb }}^{d}(X ; \mathbb{F})=\oplus_{(g) \in T} H^{d-2 \iota(g)}\left(X_{(g)} ; \mathbb{F}\right) .
$$

Definition: Let $X$ be a closed complex orbifold. We define, for $0 \leq p, q \leq$ $\operatorname{dim}_{\mathbb{C}} X$, orbifold Dolbeault cohomology groups

$$
H_{\text {orb }}^{p, q}(X ; \mathbb{C})=\oplus_{(g)} H^{p-\iota_{(g)}, q-\iota_{(g)}}\left(X_{(g)} ; \mathbb{C}\right) .
$$

Remark: When $X$ is a closed Kahler orbifold (so is each $X_{(g)}$ ), these Dolbeault groups are related to the singular cohomology groups of $X$ and $X_{(g)}$ as in the manifold case, and the Hodge decomposition theorem holds for these cohomology groups.

Definition: We define orbifold Hodge numbers by $h_{\text {orb }}^{p, q}(X)=\operatorname{dim} H_{\text {orb }}^{p, q}(X ; \mathbb{C})$.

\section{Facts from Toric Geometry}

\subsection{Fan, Orbits and Divisors}

A complex $n$-dimensional toric variety is constructed from an $n$-dimensional lattice $N$ and a fan $\Xi$ in $N$. $\Xi$ is a collection of strongly convex rational polyhedral cones $\sigma$ in the real vector space $N_{\mathbb{R}}=N \otimes_{\mathbb{Z}} \mathbb{R}$, satisfying the conditons: every face of a cone in $\Xi$ is also a cone in $\Xi$, and the intersection of two cones in $\Xi$ is a face of each. We shall always assume that $\Xi$ is finite.

Let $M=\operatorname{Hom}(N, \mathbb{Z})$ denote the dual lattice, with dual pairing denoted by $\langle$,$\rangle . If \sigma$ is a cone in $N$, the dual cone $\check{\sigma}$ is the set of vectors in $M_{\mathbb{R}}$ that are nonnegative on $\sigma$. This determines a commutative semigroup $S_{\sigma}=\check{\sigma} \cap M=\{u \in M:\langle u, v\rangle \geq 0$ for all $v \in \sigma\}$. This semigroup is finitely generated. The corresponding group-algebra $\mathbb{C}\left[S_{\sigma}\right]$ which is the $\mathbb{C}$-algebra with generators $\chi^{m}$ for each $m \in S_{\sigma}$ and relations $\chi^{m} \chi^{m^{\prime}}=\chi^{m+m^{\prime}}$, is a finitely generated commutative $\mathbb{C}$-algebra. Such an algebra corresponds to an $n$-dimensional affine variety $U_{\sigma}:=\operatorname{spec} \mathbb{C}\left[S_{\sigma}\right]$. 
If $\tau$ is a face of $\sigma$, then $S_{\sigma}$ is contained in $S_{\tau}$. So $\mathbb{C}\left[S_{\sigma}\right]$ is a subalgebra of $\mathbb{C}\left[S_{\tau}\right]$. This gives a map $U_{\tau} \rightarrow U_{\sigma}$. In fact, $U_{\tau}$ is a principal open subset of $U_{\sigma}$ : if we choose $u \in S_{\sigma}$ so that $\tau=\sigma \cap u^{\perp}$, then $U_{\tau}=\left\{x \in U_{\sigma}: u(x) \neq 0\right\}$. With these identifications, these affine varieties for different cones fit together to form an algebraic variety $X_{\Xi}$. We will write $X$ for $X_{\Xi}$ when there is no confusion. $X$ is a normal Cohen-Macaulay variety.

The properties of $\Xi$ strongly affect the geometry of $X$. For example:

(a) $X$ is complete(i.e., compact) iff the support $|\Xi|:=\cup_{\sigma \in \Xi} \sigma=N_{\mathbb{R}}$.

(b) $X$ is nonsingular iff for every cone in $\Xi$, its 1-dimensional generators (defined below) are part of a $\mathbb{Z}$-basis of $N$. Such a fan is called smooth.

(c) $X$ is an orbifold iff the generators for every cone in $\Xi$ are linearly independent over $\mathbb{R}$. We say $X$ and $\Xi$ are simplicial.

The affine variety corresponding to the trivial cone $\{0\}$ is the torus $T_{N}=$ $N \otimes \mathbb{C}=\operatorname{Spec} \mathbb{C}[M]$. It is an affine (Zariski) open subset of $X_{\Xi}$. The action of $T_{N}$ on itself extends to an algebraic action of $T_{N}$ on $X_{\Xi}$. This action has exactly one orbit corresponding to each cone $\tau \in \Xi: O_{\tau}=\operatorname{Hom}\left(\tau^{\perp} \cap M, \mathbb{C}^{*}\right)$. We write $\tau<\sigma$ if $\tau$ is a face of $\sigma$ and we write $\overline{O_{\sigma}}$ for the closure of $O_{\sigma}$ in $X$. Then

(a) $U_{\sigma}=\bigsqcup_{\tau<\sigma} O_{\tau}$; [Note $\tau=\sigma$ is included]

(b) $\overline{O_{\tau}}=\bigsqcup_{\gamma>\tau} O_{\gamma}$;

(c) $X_{\Xi}=\bigsqcup_{\tau \in \Xi} O_{\tau}$;

For each $d, \Xi(d)$ denotes the set of $d$ dimensional cones of $\Xi$. We reserve the letter $\eta$ to stand for elements of $\Xi(1)$. For each $\eta$, let $v_{\eta}$ denote the unique generator of the semigroup $\eta \cap N$. Using these generators, a cone $\sigma \in \Xi$ can be written $\sigma=\left\{\sum_{\eta \subset \sigma} a_{\eta} v_{\eta}: a_{\eta} \geq 0\right\}$. The $v_{\eta} \in \sigma$ are the generators of $\sigma$. A standard abuse of notation is to identify $\eta$ with $v_{\eta}$. If $r=|\Xi(1)|$ is the number of 1-dimensional cones, we sometimes write the $v_{\eta}$ 's as $v_{1}, \ldots, v_{r}$.

Each $\overline{O_{\eta}}$ is an irreducible $T_{N}$-invariant Weil divisor denoted $D_{\eta}$. Furthermore, $m \in M$ gives a character $\chi^{m}: T_{N} \rightarrow \mathbb{C}^{*}$, and regarding $\chi^{m}$ as a rational function on $X$, we have $\operatorname{div}\left(\chi^{m}\right)=\sum_{\eta}\left\langle m, v_{\eta}\right\rangle D_{\eta}$. We will always assume that the 1-dimensional cones span $N_{\mathbb{R}}$. Then the Chow group $A_{n-1}(X)$ of Weil divisors modulo linear equivalence can be computed directly from the fan . By using the above, we get an exact sequence 


$$
0 \longrightarrow M \longrightarrow \mathbb{Z}^{\Xi(1)} \longrightarrow A_{n-1}(X) \longrightarrow 0
$$

where $m \in M$ maps to $\left(. .,\left\langle m, v_{\eta}\right\rangle, ..\right) \in \mathbb{Z}^{\Xi(1)}$ and $\left(. ., a_{\eta}, ..\right) \in \mathbb{Z}^{\Xi(1)}$ maps to the divisor class of $\sum_{\eta} a_{\eta} D_{\eta}$. Thus $A_{n-1}(X)$ has rank $r-n$. A Weil divisor $D=\sum_{\eta} a_{\eta} D_{\eta}$ is Cartier iff for each $\sigma \in \Xi$, there is $m_{\sigma} \in M$ such that $\left\langle m_{\sigma}, v_{\eta}\right\rangle=-a_{\eta}$ whenever $\eta \subset \sigma$. Polytopes arise naturally when dealing with toric varieties. If $X$ is complete and $D=\sum_{\eta} a_{\eta} D_{\eta}$ is Cartier, then

$\Delta_{D}=\left\{m \in M_{\mathbb{R}}:\langle m, v\rangle \geq \phi_{D}(v) \forall v \in N_{\mathbb{R}}\right\}$

$=\left\{m \in M_{\mathbb{R}}:\langle m, v\rangle \geq-a_{\eta} \forall \eta\right\}$ is a polytope.

There is a $T_{N}$-equivariant map $H^{0}(X, \mathcal{O}(D)) \simeq \bigoplus_{m \in \Delta_{D} \cap M} \mathbb{C} \chi^{m}$.

To see why this is true, think of sections of $\mathcal{O}(D)$ as rational functions $f$ on $X$ such that $\operatorname{div}(f)+D \geq 0 . \operatorname{Div}\left(\chi^{m}\right)+D \geq 0$ is equivalent to $\left\langle m, v_{\eta}\right\rangle \geq-a_{\eta} \forall \eta$ and the isomorphism follows.

A cartier divisor $D$ is ample $\Longleftrightarrow\left\langle m_{\sigma}, v_{\eta}\right\rangle>-a_{\eta}$ whenever $\eta$ is not in $\sigma$ and $\sigma$ is $n$-dimensional.

$\Longleftrightarrow m_{\sigma} \neq m_{\tau}$ for $\sigma \neq \tau$ in $\Xi(n)$ and $\Delta_{D}$ is an $n$-dimensional polytope with vertices $\left\{m_{\sigma}: \sigma \in \Xi(n)\right\}$.

A polytope is called integral if its vertices are integral. $\Delta_{D}$ is integral if $D$ is ample.

\subsection{Projective Toric variety from polytope}

Given any $n$-dimensional integral polytope $\Delta$ one can canonically associate a $\mathbb{C}$-algebra $S_{\Delta}$ to it. See $[[6]$,section 3.2 .2$]$ for details. Then the projective variety $\mathbb{P}_{\Delta}:=\operatorname{Proj}\left(S_{\Delta}\right)$ turns out to be a toric variety with a fan called the normal fan of $\Delta$. We will describe the normal fan in the special case of Fano toric varieties later.

The $T_{N}$ orbit closures of $\mathbb{P}_{\Delta}$ are in one-to-one correspondence with the nonempty faces $F$ of $\Delta$. There is a canonical surjection of polytope rings $\pi_{F}: S_{\Delta} \rightarrow S_{F}$ which induces a natural inclusion of toric varieties $\mathbb{P}_{F} \hookrightarrow \mathbb{P}_{\Delta}$. $\mathbb{P}_{\Delta}$ comes with a specific choice of ample divisor $D_{\Delta}$ such that $\Delta_{D_{\Delta}}=\Delta$. 
Choose a basis for $M$. This corresponds to picking coordinates $t_{1}, \ldots, t_{n}$ for the torus $T_{N}$. Then, if $m \in M$ is written $m=\left(a_{1}, . ., a_{n}\right)$, we have $\chi^{m}=\prod_{i=1}^{n} t_{i}^{a_{i}}$, so we can write $t^{m}$ instead of $\chi^{m}$. For any $k \geq 0$, we have the space of Laurent polynomials $L(k \Delta)=\left\{f: f=\sum_{m \in k \Delta \cap M} \lambda_{m} t^{m}, \lambda_{m} \in \mathbb{C}\right\}$. Each $f \in L(k \Delta)$ gives the affine hypersurface $Z_{f} \subset T_{N}$ defined by $f=0$. $L(k \Delta) \simeq H^{0}\left(\mathbb{P}_{\Delta}, \mathcal{O}_{\mathbb{P}_{\Delta}}\left(k D_{\Delta}\right)\right)$. Under this isomporphism, $f$ corresponds to an effective divisor $\bar{Z}_{f} \subset \mathbb{P}_{\Delta}$. $\bar{Z}_{f}$ is a compactification of $Z_{f}$ and is exactly the hypersurface of $\mathbb{P}_{\Delta}$ corresponding to $f$. We will use the following notation:

(a) $l(k \Delta)=|k \Delta \cap M|=\operatorname{dim}(L(k \Delta))$

(b) $l^{*}(k \Delta)=\mid\{m \in k \Delta \cap M: m$ is not in any facet of $k \Delta \cap M\} \mid$

\subsection{Homogeneous coordinate ring}

We introduced coordinates $t_{1}, . ., t_{n}$ on the torus of a toric variety. But it is useful to have global coordinates, similar to homogeneous coordinates on projective space. If $X$ is given by a fan $\Xi$ in $N_{\mathbb{R}}$, we introduce a variable $x_{\eta}$ for each $\eta \in \Xi(1)$ and consider the polynomial ring $S=\mathbb{C}\left[x_{\eta}: \eta \in \Xi(1)\right]$. A monomial in $S$ is written $x^{D}=\prod_{\eta} x_{\eta}^{a_{\eta}}$, where $D=\sum_{\eta} a_{\eta} D_{\eta}$ is an effective torus-invariant divisor on $X$. We say that $x^{D}$ has degree $\operatorname{deg}\left(x^{D}\right)=[D] \in$ $A_{n-1}(X)$.

Thus, $S$ is graded by the Chow group $A_{n-1}(X)$. Given a divisor class $\alpha \in A_{n-1}(X), S_{\alpha}$ denotes the graded piece of $S$ of degree $\alpha$. We often write the variables as $x_{1}, \ldots, x_{r}$, where $x_{i}$ corresponds to the cone generator $v_{i}$ and $r=|\Xi(1)|$. Then $S=\mathbb{C}\left[x_{1}, \ldots, x_{r}\right]$. The ring $S$, together with the grading defined above is called the homogeneous coordinate ring of $X$. We refer the reader to [[6],chapter 3.2] for a discussion on reconstructing the toric variety starting from the homogeneous coordinate ring.

If $\tau$ is any cone of $\Xi$ then the orbit closure $\overline{O_{\tau}}$ is given by the ideal $\left(x_{i}: i\right.$ such that $v_{i}$ is a generator of $\tau$ ) of $S$. Also the graded pieces of $S$ have nice cohomological interpretation. We noted that $L(\Delta) \simeq H^{0}\left(\mathbb{P}, \mathcal{O}_{\mathbb{P}}\left(D_{\Delta}\right)\right)$. Now the map sending the Laurent monomial $t^{m}$ to $\prod_{\eta} x_{\eta}^{\left\langle m, v_{\eta}\right\rangle+a_{\eta}}$ induces an isomorphism $H^{0}\left(X, \mathcal{O}_{X}(D)\right) \simeq S_{\alpha}$, where $\alpha=[D] \in A_{n-1}(X)$. 


\subsection{Fano toric Varieties}

First we note that for an arbitrary toric variety $X$, the anticanonical divisor $-K_{X}=\sum_{\eta} D_{\eta}$. The following may be taken as definitions.

Definition: A toric variety $X$ is Gorenstein iff $-K_{X}$ is Cartier. A complete toric variety $X$ is Fano iff $-K_{X}$ is Cartier and ample.

The anticanonical divisor of a Fano toric variety $X$ detemines an integral polytope $\Delta$ with special properties :

(a) All facets $\Gamma$ of $\Delta$ are supported by an affine hyperplane of the form $\left\{m \in M_{\mathbb{R}}:\left\langle m, v_{\Gamma}\right\rangle=-1\right\}$ for some $v_{\Gamma} \in N$.

(b) $\operatorname{Int}(\Delta) \cap M=0$.

Definition: Such a polytope is called reflexive.

The polar polytope $\Delta^{\circ}$ of the reflexive polytope $\Delta$ is obtained by $\Delta^{\circ}=$ $\left\{v \in N_{\mathbb{R}}:\langle m, v\rangle \geq-1\right.$ for all $\left.m \in \Delta\right\} \subset N_{\mathbb{R}}$. The fan $\Xi$ of $X$ can be retrieved by coning over the proper faces of $\Delta^{\circ}$. This fan is called the normal fan of $\Delta$ and $X=\mathbb{P}_{\Delta} . \Delta^{\circ}$ is also reflexive and $\left(\Delta^{\circ}\right)^{\circ}=\Delta$. The Fano troic variety constructed from the normal fan of $\Delta^{\circ}$ is denoted by $\mathbb{P}_{\Delta^{\circ}}$.

We shall use $F$ and $F^{\circ}$ to denote a face of $\Delta$ and $\Delta^{\circ}$ respectively. There exists an inclusion reversing duality between the faces of $\Delta$ and $\Delta^{\circ}$. For instance, the face of $\Delta$ which is dual to the face $F^{\circ}$ of $\Delta^{\circ}$ is defined as $\widehat{F^{\circ}}:=\left\{m \in \Delta:\langle n, m\rangle=-1 \forall n \in F^{\circ}\right\}$. If $\tau$ is the cone in the normal fan of $\Delta$ associated to the face $F^{\circ}$, then the orbit closure $\bar{O}_{\tau}=\mathbb{P}_{\widehat{F^{\circ}}}$.

Generic anticanonical hypersurfaces $V$ in $\mathbb{P}_{\Delta}$ and $V^{\circ}$ in $\mathbb{P}_{\Delta^{\circ}}$ constitute mirror families of Calabi-Yau varieties. These varieties are orbifolds if the corresponding ambient toric variety is simplicial. Observe that $V$ can be identified with an element of $L(\Delta)$.

A 'maximal projective subdivision' of the fan of the toric variety gives a maximal projective crepant partial (MPCP) resolution [see [1] or [6]] of the hypersurface by taking proper transforms. Let $\widehat{V}$ and $\widehat{V^{\circ}}$ denote the MPCP resolutions of $V$ and $V^{\circ}$ respectively. These are again Calabi-Yau. These are smooth if $n=4$.

Remark: A gorenstein toric variety or a Calabi-Yau hypersurface of a 
Fano toric variety has gorenstein canonical singularities. In particular, the degree shifting numbers are integers. Moreover, the singularity locus has at least complex codimension two.

\section{Twisted Sectors}

We claim that the twisted sectors of a toric variety or a Calabi-Yau hypersurface can be identified with suborbifolds. Note that in general a twisted sector could be a multiple cover of the corresponding singular locus even if the group actions are all abelian. The situation is simpler in toric case because there exist canonical local coordinates and uniformizing systems such that the group actions are in fact diagonal. They can be easily chased around to prove the claim.

\subsection{Twisted Sectors in Simplicial Toric Variety}

Let $\Xi$ be any simplicial fan. Then the orbifold structure of the toric variety $X_{\Xi}$ can be descibed as follows. Let $\sigma$ be any $\mathrm{n}$ dimensional cone of $\Xi$. Let $v_{1}, \ldots, v_{n}$ be the primitive 1 dim generators of $\sigma$. These are linearly independent in $N_{\mathbb{R}}$. Let $N_{\sigma}$ be the sublattice of $N$ generated by $v_{1}, . ., v_{n}$. Let $G_{\sigma}:=N / N_{\sigma}$ be the quotient group. $G_{\sigma}$ is finite and abelian.

Let $\sigma^{\prime}$ be the cone $\sigma$ regarded in $N_{\sigma}$. Let $\tilde{\sigma}^{\prime}$ be the dual cone of $\sigma^{\prime}$ in $M_{\sigma}$, the dual lattice of $N_{\sigma} . U_{\sigma^{\prime}}=\operatorname{spec}\left(\mathbb{C}\left[\check{\sigma^{\prime}} \cap M_{\sigma}\right]\right)$. Note that $\sigma^{\prime}$ is a smooth cone in $N_{\sigma}$. So $U_{\sigma^{\prime}} \cong \mathbb{C}^{n}$.

There is a canonical dual pairing $M_{\sigma} / M \times N / N_{\sigma} \rightarrow \mathbb{Q} / \mathbb{Z} \rightarrow \mathbb{C}^{*}$, the first map by the pairing $\langle$,$\rangle and the second by q \mapsto \exp (2 \pi i q)$. Now $G_{\sigma}$ acts on $\mathbb{C}\left[M_{\sigma}\right]$, the group ring of $M_{\sigma}$, by : $v\left(\chi^{u}\right)=\exp (2 \pi i\langle u, v\rangle) \chi^{u}$, for $v \in N$ and $u \in M_{\sigma}$. Note that

$\left(\mathbb{C}\left[M_{\sigma}\right]\right)^{G_{\sigma}}=\mathbb{C}[M]$

Thus $G_{\sigma}$ acts on $U_{\sigma^{\prime}}$. Let $\pi_{\sigma}$ be the quotient map. Then $U_{\sigma}=U_{\sigma^{\prime}} / G_{\sigma}$. So $U_{\sigma}$ is uniformised by $\left(U_{\sigma^{\prime}}, G_{\sigma}, \Pi_{\sigma}\right)$. For any $\tau<\sigma$, the orbifold structure on $U_{\tau}$ is same as the one induced from the uniformising system on $U_{\sigma}$. Then by the description of the toric gluing it is clear that $\left\{\left(U_{\sigma^{\prime}}, G_{\sigma}, \Pi_{\sigma}\right): \sigma \in \Xi(n)\right\}$ defines a reduced orbifold structure on $X$. We give a more explicit verification of this fact below. 
Let $\mathcal{B}$ be the nonsingular matrix with generators $v_{1}, . ., v_{n}$ of $\sigma$ as rows. Then $\tilde{\sigma}^{\prime}$ is generated in $M_{\sigma}$ by the the column vectors $v^{1}, . ., v^{n}$ of the matrix $\mathcal{B}^{-1}$. So $\chi^{v^{1}}, . ., \chi^{v^{n}}$ are the coordinates of $U_{\sigma^{\prime}}$. For any $\kappa=\left(k_{1}, . ., k_{n}\right) \in N$, the corresponding coset $[\kappa] \in G_{\sigma}$ acts on $U_{\sigma^{\prime}}$ in these coordinates as a diagonal matrix: $\operatorname{diag}\left(\exp ^{2 \pi i c_{1}}, \ldots, \exp ^{2 \pi i c_{n}}\right)$ where $c_{i}=\left\langle\kappa, v^{i}\right\rangle$. Such a matrix is uniquely represented by an $n$-tuple $a=\left(a_{1}, . ., a_{n}\right)$ where $a_{i} \in[0,1)$ and $c_{i}=a_{i}+b_{i}, b_{i} \in \mathbb{Z}$. In matrix notation, $\kappa \mathcal{B}^{-1}=a+b \Longleftrightarrow \kappa=a \mathcal{B}+b \mathcal{B}$. We denote the inetgral vector $a \mathcal{B}$ in $N$ by $\kappa_{a}$ and the diagonal matrix corresponding to $a$ by $g_{a} . \kappa_{a} \leftrightarrow g_{a}$ gives a one to one correspondence between the elements of $G_{\sigma}$ and the integral vectors in $N$ that are linear combinations of the generators of $\sigma$ with cofficients in $[0,1)$.

Now let us examine the orbifold chart induced by $\left(U_{\sigma^{\prime}}, G_{\sigma}, \pi_{\sigma}\right)$ at any point $x \in U_{\sigma}$. By the orbit decomposition, there is unique $\tau \in \sigma$ such that $x \in O_{\tau}$. Wlog assume $\tau$ is generated by $v_{1}, . ., v_{j}, j \leq n$. Then any preimage of $x$ with respect to $\pi_{\sigma}$ has coordinates $\chi^{v^{i}}=0$ iff $i \leq j$. Let $z=\left(0, . ., 0, z_{j+1}, . ., z_{n}\right)$ be one such preimage. Let $G_{\tau}:=\left\{g_{a} \in G_{\sigma}: a_{i}=0\right.$ if $j+1 \leq i \leq n\}$. We can find a small neighbourhood $W \subset\left(\mathbb{C}^{*}\right)^{n-j}$ of $\left(z_{j+1}, . ., z_{n}\right)$ such that the inclusions $\mathbb{C}^{j} \times W \hookrightarrow U_{\sigma^{\prime}}$ and $G_{\tau} \hookrightarrow G_{\sigma}$ induces an injection of uniformising systems $\left(\mathbb{C}^{j} \times W, G_{\tau}, \pi\right) \hookrightarrow\left(U_{\sigma^{\prime}}, G_{\sigma}, \pi_{\sigma}\right)$ on some small open neighbourhood $U_{x}$ of $x$. So we have $G_{x}=G_{\tau}$ and an orbifold chart $\left(\mathbb{C}^{j} \times W, G_{\tau}, \pi\right)$. Note that $G_{\tau}$ can be constructed from the set $\left\{\kappa_{a}=\sum_{i=1}^{j} a_{i} V_{i}: \kappa_{a} \in N, a_{i} \in[0,1)\right\}$ which is completely determined by $\tau$ and hence is independent of $\sigma$.

Now we determine the twisted sectors. Take any $x \in X . x$ belongs to a unique $O_{\tau}$. Wlog the generators of $\tau$ are $v_{1}, . ., v_{j}$. Consider any $n$ dimensional $\sigma>\tau$. Wlog $v_{1}, . ., v_{n}$ generates $\sigma$. Pick $g_{a}$ in $G_{x}$ such that $a_{i} \neq 0, \forall i \leq j$ i.e, $\kappa_{a}$ lies in the interior of $\tau$. We want to find the twisted sector $X_{\left(g_{a}\right)}$. Consider $g_{a}$ as an element of $G_{\sigma}$. It is clear that $g_{a}$ fixes $z \in U_{\sigma^{\prime}}$ iff $z_{1}=. .=z_{j+s}=0$, for some $s \geq 0$ i.e., $\pi_{\sigma}(z) \in O_{\tau}$ or $\pi_{\sigma}(z) \in O_{\delta}$ for some $\delta>\tau$. So $X_{\left(g_{a}\right)} \cap U_{\sigma}=\bar{O}_{\tau} \cap U_{\sigma}$. Since a twisted sector is connected, $X_{\left(g_{a}\right)}=\bar{O}_{\tau}$. If $g_{a} \in G_{x}$ is such that (wlog) only $a_{1}, . ., a_{k} \neq 0, k<j$, then $g_{a} \in G_{\delta}$ where $\delta$ is the cone generated by $v_{1}, . ., v_{k}$ and by the above argument $X_{\left(g_{a}\right)}=\bar{O}_{\delta}$. Thus we have proved the following theorem.

Theorem 1 A twisted sector of any simplicial toric variety $X_{\Xi}$ is isomorphic 
to a subvariety $\bar{O}_{\tau}$ of $X_{\Xi}$ for some cone $\tau \in \Xi$. Moreover, there is a one-toone correspondence between the set of twisted sectors of the type $\overline{O_{\tau}}$ and the set of integral vectors in the interior of $\tau$ which are linear combinations of the 1-dimensional generators of $\tau$ with coeffients in $(0,1)$.

Note that the degree shifting number $\iota_{\left(g_{a}\right)}=\sum a_{i}$. Now if $X_{\Xi}$ is Fano, i.e., $\Xi$ is obtained by coning over the faces of a reflexive polytope $\Delta^{\circ}$, then the twisted sectors with $\iota=1$ are in one to one correspondence with the integral interior points of faces of $\Delta^{\circ}$.

\subsection{Twisted sectors of hypersurface of a Fano variety}

We identify the twisted sectors of a generic nondegenerate anticanonical (Calabi-Yau) hypersurface $V$ of a simplicial Fano toric variety $X=\mathbb{P}_{\Delta}$. Nondegenerate means that $V \cap O_{\tau}$ is either empty or a smooth subvariety of codimension one in $O_{\tau}$, for each torus orbit $O_{\tau}$ in $X$. Then $V$ turns out to be a suborbifold of $X$. Also nondegeneracy is a generic condition. For a different treatment on these, see [3]. We show that $V=\bar{Z}_{f}$, for a generic $f \in L(\Delta)$, is nondegenerate and a suborbifold of $X$.

Consider any $n$-dimensional cone $\sigma$ with generators $v_{1}, \ldots, v_{n}$. For notational simplicity set $\chi^{v^{i}}=z_{i}$. Then $z_{1}, . ., z_{n}$ are the coordinates of $U_{\sigma^{\prime}}$. Let $Y$ be the preimage of $V \cap U_{\sigma}$ in $U_{\sigma^{\prime}}$ with respect to $\pi_{\sigma}$. Then $Y$ is defined by the equation $\sum_{m \in \Delta \cap M} \lambda_{m} \prod_{i=1}^{n} z_{i}{ }^{\left\langle m, v_{i}\right\rangle+1}=0$. This is because, $t^{m}=\prod_{i=1}^{n} z_{i}^{q_{i}} \Longleftrightarrow m=\sum q_{i} v^{i}=\mathcal{B}^{-1} q \Longleftrightarrow q=m \mathcal{B} \Longleftrightarrow q_{i}=\left\langle m, v_{i}\right\rangle$. The one is added to ensure that $V$ is anticanonical. Note that by definition of $\Delta,\left\langle m, v_{i}\right\rangle+1 \geq 0$. If $\lambda_{m_{\sigma}} \neq 0$ then $Y$ does not pass through the origin. It can be checked from this description using Bertini's theorem that for generic values of the coefficients $\lambda_{m}, Y$ is a smooth submanifold of $U_{\sigma^{\prime}}$ that intersects the coordinate planes $z_{i_{1}}=. .=z_{i_{j}}=0$ transversely.

$Y$ is $G_{\sigma}$-stable by (4.1.1). When $Y$ is smooth, all singularities of $V \cap U_{\sigma}$ are quotient singularities induced by action of $G_{\sigma}$ on $Y$. Since there are only finitely many $n$-dimensional cones, $V$ is nondegenerate and a suborbifold of $X$. $\left(Y, G_{\sigma}, \pi_{\sigma}\right)$ is an uniformizing system for $V \cap U_{\sigma}$. Let $\tau$ be the face of $\sigma$ obtained by coning over the face $F^{\circ}$ of $\Delta^{\circ}$. Wlog let $v_{1}, . ., v_{j}: j \geq 2$ be the generators of $\tau$. (We remarked earlier in section 3.4 that the Gorenstein 
condition rules out codimension one singularities.) We want to find a chart for any point $x \in V \cap O \tau$. By our earlier remark that $Y$ misses the origin of $U_{\sigma^{\prime}}, V \cap \bar{O}_{\sigma}$ is empty. So we need only consider proper faces of $\sigma$. By a result of Fulton[[8],section 5.3], $V \cap \bar{O}_{\tau}$ consists of $l^{*}\left(\widehat{F^{\circ}}\right)+1$ points if $F^{\circ}$ is a codimension 2 face of $\Delta^{\circ}$ i.e., $j=n-1 \Longleftrightarrow \operatorname{dim} O_{\tau}=1$. Since the the only other points in $\overline{O_{\tau}}$ in this case are $O_{\sigma}$ for $n$-dimensional cones $\sigma>\tau$, all the intersection points actually lie in $O_{\tau}$. If codimension $F^{\circ}$ is bigger than 2, then again by Bertini $V \cap \bar{O}_{\tau}$ is irreducible.

Following the same notation as before, $x$ has a small neighborhood $U_{x} \cap Y$ such that $\left(\left(\mathbb{C}^{j} \times W\right) \cap Y, G_{\tau}, \pi\right)$ is a chart for $V$ at $x$. $\mathbb{C}^{j} \times W$ is , as before, a suitable neighbourhood of some preimage $z$ of $x$ in $U_{\sigma^{\prime}}$. The tangent space $T Y_{z}$ is a $G_{\tau}$-stable subspace of $T \mathbb{C}_{z}^{n}$. Any $g_{a} \in G_{\tau}$ acts trivially on $T W_{z}=\operatorname{span}\left\{\partial / \partial z_{i}, i=j+1, \ldots, n\right\}$. By transversality, we can choose basis $\left\{\xi_{1}, . ., \xi_{n}\right\}$ of $T \mathbb{C}_{z}^{n}$ such that $\xi_{i} \in T Y_{z} \forall i \leq n-1$ and $\xi_{n} \in T W_{z}$. $g_{a}$ acts trivially on $\xi_{n}$. This implies that the degree shifting number of $\left.g_{a}\right|_{T Y_{z}}$ is still $\sum_{i=1}^{j} a_{i}$

From the description of the charts, it is clear that twisted sectors of $V$ are isomorphic to $V \cap \overline{O_{\tau}}$ where $2 \leq \operatorname{dim}(\tau) \leq n-1$. Recall that $\bar{O}_{\tau}=\mathbb{P}_{\widehat{F^{\circ}}}$ where $\widehat{F^{\circ}}$ is the face of $\Delta$ dual to $F^{\circ}$. In particular we have the following theorem.

Theorem 2 Let $V$ be a generic nondegenerate anticanonical hypersurface of an $n$-dimensional simplicial Fano toric variety $\mathbb{P}_{\Delta}$. Then the twisted sectors of $V$ are isomorphic to $V \cap \mathbb{P}_{\widehat{F^{\circ}}}$ for some face $F^{\circ}$ of $\Delta^{\circ}$ such that $1 \leq \operatorname{dim} F^{\circ} \leq n-2$. There is exactly one twisted sector of this type having $\iota=1$, corresponding to each integral interior point of $F^{\circ}$ if $\operatorname{dim} F^{\circ}<n-2$. If $\operatorname{dim} F^{\circ}=n-2$, then there are exactly $l^{*}\left(\widehat{F^{\circ}}\right)+1$ twisted sectors of this type having $\iota=1$, corresponding to each integral interior point of $F^{\circ}$.

\section{Orbifold Hodge Numbers}

\section{$5.1 h_{\text {orb }}^{1,1}(V)$}

Let $V_{(g)}$ denote a twisted sector of the hypersurface $V$ and $\iota_{(g)}$ its degree shifting number. 
$h_{\text {orb }}^{1,1}(V)=h^{1,1}(V)+\sum_{\iota_{(g)}=1} h^{0,0}\left(V_{(g)}\right)$. Since $h^{0,0}\left(V_{(g)}\right)=1$ for each twisted sector, by theorem (4.2) we obtain

$$
\begin{aligned}
& \sum_{\iota_{(g)}=1} h^{0,0}\left(V_{(g)}\right) \\
= & \sum_{1 \leq \operatorname{dim}\left(F^{\circ}\right) \leq n-2} l^{*}\left(F^{\circ}\right)+\sum_{\operatorname{dim}\left(F^{\circ}\right)=n-2} l^{*}\left(F^{\circ}\right) l^{*}\left(\widehat{F^{\circ}}\right) \\
= & l\left(\Delta^{\circ}\right)-r-1-\sum_{\operatorname{dim}\left(F^{\circ}\right)=n-1} l^{*}\left(F^{\circ}\right)+\sum_{\operatorname{dim}\left(F^{\circ}\right)=n-2} l^{*}\left(F^{\circ}\right) l^{*}\left(\widehat{F^{\circ}}\right)
\end{aligned}
$$

To compute $h^{1,1}(V)$ we invoke the following Lefschetz hyperplane theorem [[3],Proposition 10.8] .

Lemma 1 Let $V$ be a nondegenerate ample hypersurface of an $n$-dimensional complete simplicial toric variety $X$. Then the natural map, induced by inclusion, $j^{*}: H^{i}(X) \rightarrow H^{i}(V)$ is an isomorphism for $i<n-1$ and an injection for $i=n-1$.

In our case $V$ is anticanonical, and since the anticanonical divisor of a Fano variety is ample, $V$ is ample. Also it is well known[[8],section 5.1] that for any simplicial toric variety $X, H^{2}(X, \mathbb{R})=H^{1,1}(X, \mathbb{R})=A_{n-1}(X) \otimes \mathbb{R}$. So for $n \geq 4, h^{1,1}(V)=h^{1,1}\left(\mathbb{P}_{\Delta}\right)=\operatorname{rank} A_{n-1}\left(\mathbb{P}_{\Delta}\right)=r-n$. Thus we have the following theorem.

Theorem 3 For any generic nondegenerate anticanonical hypersurface $V$ of an $n$-dimensional simplicial Fano toric variety $\mathbb{P}_{\Delta}, n \geq 4$,

$$
h_{\text {orb }}^{1,1}(V)=l\left(\Delta^{\circ}\right)-n-1-\sum_{\operatorname{dim}\left(F^{\circ}\right)=n-1} l^{*}\left(F^{\circ}\right)+\sum_{\operatorname{dim}\left(F^{\circ}\right)=n-2} l^{*}\left(F^{\circ}\right) l^{*}\left(\widehat{F^{\circ}}\right) .
$$

\section{$5.2 h^{n-2,1}(V)$}

Next we compute $h^{n-2,1}(V)$ for $n \geq 4$. For this we have to use the homogeneous coordinate ring $S$ of $X=\mathbb{P}_{\Delta}$. Let $v_{1}, . ., v_{r}$ be the one dimensional cones of the normal fan of $\Delta$. Let $x_{1}, . ., x_{r}$ be the corresponding homogeneous coordinates. Let $\beta_{0}=\left[-K_{X}\right]=\left[\sum_{i=1}^{r} D_{i}\right] \in A_{n-1}(X)$. Then $S_{\beta_{0}} \simeq L(\Delta)$. And the divisor $f \in L(\Delta)$ corresponding to $V$ can be written in the homogeneous coordinates as $f=\sum_{m \in M \cap L(\Delta)} \lambda_{m} \prod_{i=1}^{r} x_{i}{ }^{\left\langle m, v_{i}\right\rangle+1}$. For notational simplicity, we will denote $\prod_{i=1}^{r} x_{i}{ }^{\left\langle m, v_{i}\right\rangle+1}$ by $\mathbf{x}^{m}$ for any $m \in M$. Define the Jacobian ideal of $f$ to be $J(f)=\left\langle\partial f / \partial x_{1}, \ldots, \partial f / \partial x_{r}\right\rangle$.

First we quote the following theorem [[3], theorem 10.13] . 
Lemma 2 Let $X$ be an d-dimensional complete simplicial toric variety and $V \subset X$ be a quasi-smooth(i.e.,suborbifold) ample hypersurface defined by $f \in S_{\beta}$. Then for $k \neq(d / 2)+1$, there exists a canonical isomorphism

$(S / J(f))_{k \beta-\beta_{0}} \simeq P H^{d-k, k-1}(V)$.

Remark: The primitive cohomolgy $P H^{d-1}(V):=H^{d-1}(V) /\left(\operatorname{im} H^{d-1}(X)\right.$. This coincides with the usual cohomology if $d$ is even.

For our application of this lemma: $d=n, k=2$ and $\beta=\beta_{0}$. So we need to compute the rank of $(S / J(f))_{\beta_{0}}$. Also, $H^{n-2,1}(X)=0$ if $n \geq 4$, so that $H^{n-2,1}(V)=P H^{n-2,1}(V)$.

Lemma $3 x_{i} \partial f / \partial x_{i} \in(J(f))_{\beta_{0}}, i=1, . ., r$, and the space of complex linear relations among these has dimension $r-(n+1)$.

\section{Proof}

$$
\begin{aligned}
& x_{i} \partial f / \partial x_{i}=\sum_{m} \lambda_{m}\left(\left\langle m, v_{i}\right\rangle+1\right) \mathbf{x}^{m} . \\
& \sum_{i} c_{i} x_{i} \partial f / \partial x_{i}=\sum_{m} \lambda_{m}\left(\left\langle m, \sum_{i} c_{i} v_{i}\right\rangle+\sum_{i} c_{i}\right) \mathbf{x}^{m}
\end{aligned}
$$

For a generic $f$ we can assume that $\lambda_{m} \neq 0$ for each $m \in \Delta \cap M$. Hence $\sum_{i} c_{i} x_{i} \partial f / \partial x_{i} \equiv 0 \Longleftrightarrow\left\langle m, \sum_{i} c_{i} v_{i}\right\rangle+\sum_{i} c_{i}=0 \forall m \in \Delta \cap M$

In particular, taking $m=0$ we get $\sum_{i} c_{i}=0$. Therefore $\left\langle m, \sum_{i} c_{i} v_{i}\right\rangle=0$ $\forall m \in \Delta \cap M$ and since $\Delta$ is $n$-dimensional we have $\sum_{i} c_{i} v_{i}=0$.

Thus $\sum_{i} c_{i} x_{i} \partial f / \partial x_{i} \equiv 0 \Longleftrightarrow \sum_{i} c_{i} v_{i}=0$ and $\sum_{i} c_{i}=0$.

Now let $\tilde{v}_{i}=\left(v_{i}, 1\right) \in \mathbb{R}^{n+1} \subset \mathbb{C}^{n+1}=\mathbb{R}^{n+1} \otimes \mathbb{C}$.

Since the $v_{i}$ 's are vertices of $\Delta^{\circ}$, the $\tilde{v}_{i}$ 's are generators of the $(n+1)$ dimensional cone $\left\{q \in \mathbb{R}^{n+1}: q t \in \Delta^{\circ}\right.$ for some $\left.t \in \mathbb{R}_{>0}\right\}$. So the $\tilde{v}_{i}$ 's span $\mathbb{R}^{n+1}$ over $\mathbb{R}$, and hence they span $\mathbb{R}^{n+1} \otimes \mathbb{C}$ over $\mathbb{C}$.

Note that $\left(\sum c_{i} v_{i}, \sum c_{i}\right)=\sum c_{i} \tilde{v}_{i}$.

Hence the lemma follows.

Wlog assume that the $x_{k} \partial f / \partial x_{k}, k=1, . ., n+1$, are linearly inedendent. In other words $\tilde{v_{k}}, k=1, \ldots, n+1$ are linearly independent. We want to find monomials $\prod_{j \neq i} x_{j} p_{j}$ that have same degree in $S$ as $x_{i}$. So we want $m^{*} \in M$ such that $\left\langle m^{*}, v_{j}\right\rangle=p_{j} \geq 0$ if $j \neq i$ and $\left\langle m^{*}, v_{i}\right\rangle=-1$. Such $m^{*}$ is given by 
interior lattice points of $F_{i}$, the $(n-1)$-dimensional face of $\Delta$ that is dual to the 0 -dimensional face $\left\{v_{i}\right\}$ of $\Delta^{\circ}$.

Then for each $m^{*} \in\left(\operatorname{Int} F_{i} \cap M\right), \prod_{j \neq i} x_{j}\left\langle m^{*}, v_{j}\right\rangle \partial f / \partial x_{i}$ belongs to $(J(f))_{\beta_{0}}$. Together with the $x_{i} \partial f / \partial x_{i}$, these generate $(J(f))_{\beta_{0}}$ as we vary over all $i$.

$\prod_{j \neq i} x_{j}\left\langle m^{*}, v_{j}\right\rangle \partial f / \partial x_{i}$

$=\prod_{j=1}^{r} x_{j}{ }^{\left\langle m^{*}, v_{j}\right\rangle} x_{i} \partial f / \partial x_{i}$

$=\sum_{m \in \Delta \cap M} \lambda_{m}\left(\left\langle m, v_{i}\right\rangle+1\right) \mathbf{x}^{m+m^{*}}$

$=\sum_{m^{\prime} \in \Delta \cap M \cap\left(\Delta+m^{*}\right)} \lambda_{m^{\prime}-m^{*}}\left(\left\langle m^{\prime}-m^{*}, v_{i}\right\rangle+1\right) \mathbf{x}^{m^{\prime}}$

$=\sum_{m \in \Delta \cap M} \lambda_{m-m^{*}}\left(\left\langle m-m^{*}, v_{i}\right\rangle+1\right) I\left(m-m^{*} \in \Delta\right) \mathbf{x}^{m}$

Let $\operatorname{Int} F_{i} \cap M=\left\{m_{i, i_{s}}: 1 \leq s \leq t_{i} ; t_{i} \geq 0\right\}$.

Then $(J(f))_{\beta_{0}}=\operatorname{span}\left\{x_{k} \partial f / \partial x_{k}, \prod_{j=1}^{r} x_{j}{ }^{\left\langle m_{i_{s}}, v_{j}\right\rangle} x_{i} \partial f / \partial x_{i}: 1 \leq k \leq n+\right.$ $\left.1,1 \leq i_{s} \leq t_{i}, i=1, . ., r\right\}$. We want to find the dimension of this complex vector space. So we study the space of linear relations :

$\sum_{k} c_{k} x_{k} \partial f / \partial x_{k}+\sum_{i, i_{s}} d_{i, i_{s}} \prod_{j=1}^{r} x_{j}{ }^{\left\langle m_{i_{s}}, v_{j}\right\rangle} x_{i} \partial f / \partial x_{i} \equiv 0$

$\sum_{m}\left\{\sum_{k} c_{k} \lambda_{m}\left(\left\langle v_{k}, m\right\rangle+1\right)+\sum_{i, i_{s}} d_{i, i_{s}} \lambda_{m-m_{i, i_{s}}} I\left(m-m_{i, i_{s}} \in \Delta\right)\left(\left\langle m-m_{i, i_{s}}, v_{i}\right\rangle+\right.\right.$ 1) $\} \mathbf{x}^{m} \equiv 0$

$$
\Longleftrightarrow
$$

$\sum_{k} c_{k} \lambda_{m}\left(\left\langle v_{k}, m\right\rangle+1\right)+\sum d_{i, i_{s}} \lambda_{m-m_{i, i_{s}}} I\left(m-m_{i, i_{s}} \in \Delta\right)\left\langle m, v_{i}\right\rangle \equiv 0$ for each $m \in \Delta \cap M$, [note: $\left\langle m_{i, i_{s}}, v_{i}\right\rangle=-1$ ]

This is a system of $l(\Delta)$ number of linear equations in $\gamma=n+1+$ $\sum_{i=1}^{r} l^{*}\left(F_{i}\right)$ variables namely $c_{k}, d_{i, i_{s}}$. Note that $l(\Delta) \geq \gamma$. We shall find a nonsingular subsystem of rank $\gamma$.

To do so pick $n$ linearly independent vertices $m_{1}, \ldots, m_{n}$ of $\Delta$ and let $m_{n+1}=0$, the origin. Then from the above system we pick the equations corresponding to $m=m_{1}, \ldots, m_{n+1}$ and $m=m_{i, i_{s}}: i=1, . ., r ; 0 \leq i_{s} \leq t_{i}$. 
Denote this $\gamma \times \gamma$ system by $(* *)$. It can be written as :

$$
\left[\begin{array}{ll}
\mathbf{P} & \mathbf{A} \\
\mathbf{B} & \mathbf{Q}
\end{array}\right]\left(\begin{array}{l}
c \\
d
\end{array}\right)=\left(\begin{array}{l}
0 \\
0
\end{array}\right)
$$

where

$$
\begin{gathered}
\mathbf{P}=\left[\begin{array}{lll}
\lambda_{m_{1}}\left(\left\langle m_{1}, v_{1}\right\rangle+1\right) & \ldots & \lambda_{m_{1}}\left(\left\langle m_{1}, v_{n+1}\right\rangle+1\right) \\
\ldots & \ldots & \ldots \\
\lambda_{m_{n}}\left(\left\langle m_{n}, v_{1}\right\rangle+1\right) & \ldots & \lambda_{m_{n}}\left(\left\langle m_{n}, v_{n+1}\right\rangle+1\right) \\
\lambda_{0} & \ldots & \lambda_{0}
\end{array}\right] \\
\mathbf{Q}=\left[\begin{array}{lll}
\lambda_{m_{1,1}-m 1,1} I(.)\left(\left\langle m_{1,1}-m_{1,1}, v_{1}\right\rangle+1\right) & \ldots & \lambda_{m_{1,1}-m_{r, t_{r}}} I(.)\left(\left\langle m_{1,1}-m_{r, t_{r}}, v_{r}\right\rangle+1\right) \\
\ldots & \ldots & \ldots \\
\lambda_{m_{r, t_{r}}-m 1,1} I(.)\left(\left\langle m_{r, t_{r}}-m_{1,1}, v_{1}\right\rangle+1\right) & \ldots & \lambda_{m_{r, t_{r}}-m_{r, t_{r}}} I(.)\left(\left\langle m_{r, t_{r}}-m_{r, t_{r}}, v_{r}\right\rangle+1\right)
\end{array}\right]
\end{gathered}
$$

Observe that all the diagonal entries of $\mathbf{Q}$ are $\lambda_{0}$, and none of its offdiagonal entries has $\lambda_{0}$. Also any entry of $\mathbf{A}$ is of the form $\lambda_{m_{k}-m_{i, i_{s}}} I($.) and hence does not involve $\lambda_{0}$. Similarly an entry of $\mathbf{B}$ is of the form $\lambda_{m_{i, i_{s}}}\left(\left\langle m_{i, i_{s}}, v_{k}\right\rangle+1\right)$ and so does not have $\lambda_{0}$.

Consider the determinant of the coefficient matrix $\left[\begin{array}{ll}\mathbf{P} & \mathbf{A} \\ \mathbf{B} & \mathbf{Q}\end{array}\right]$ as a polynomial in the $\lambda$ 's. Then the term of this determinant having the highest power of $\lambda_{0}$ is $\left(\lambda_{0}\right)^{\sum l^{*}\left(F_{i}\right)} \operatorname{det} \mathbf{P}$. We will show below that $\operatorname{det} \mathbf{P}=$ nonzero constant times $\lambda_{m_{1}} \ldots \lambda_{m_{n}} \lambda_{0}$. Thus the determinant of the coeffcient matrix of the system $(* *)$ is a nontrivial polynomial in the $\lambda^{\prime}$ 's and is therefore nonzero for generic choice of the $\lambda$ 's. Hence $(J(f))_{\beta_{0}}$ has rank $\gamma$ as a complex vector space, for a generic $f \in L(\Delta)$. Since $S_{\beta_{0}} \simeq L(\Delta)$, so $(S / J(f))_{\beta_{0}}$ has rank $l(\Delta)-\gamma$, for a generic $f$.

Lemma 4 The $(n+1) \times(n+1)$ matrix $\mathbf{P}=\left(\left(P_{i, j}=\lambda_{m_{i}}\left(\left\langle m_{i}, v_{j}\right\rangle+1\right)\right)\right)$ is nonsingular for generic chioce of $\lambda$ 's.

\section{Proof}

Let $\mathbf{E}$ be the $(n+1) \times(n+1)$ matrix $\left(\left(E_{i, j}=\left(\left\langle m_{i}, v_{j}\right\rangle+1\right)\right)\right)$. Then $\operatorname{det} \mathbf{P}=\lambda_{m_{1}} \ldots \lambda_{m_{n+1}} \operatorname{det} \mathbf{E}$. 
We claim that $\mathbf{E}$ is nonsingular. Otherwise there exists a nontrivial vector $\left(c_{1}, \ldots, c_{n+1}\right)$ such that $\sum_{k=1}^{n+1} c_{k}\left(\left\langle m_{i}, v_{k}\right\rangle+1\right)=0$ for all $i=1, \ldots, n+1$. In particular, for $i=n+1$ we get $\sum_{k=1}^{n+1} c_{k}=0$. This implies $\sum_{k=1}^{n+1} c_{k}\left\langle m_{i}, v_{k}\right\rangle=0$ for all $i=1, \ldots, n$. Since $m_{1}, . ., m_{n}$ are linearly independent, this would imply that $\sum_{k=1}^{n+1} c_{k}\left\langle m, v_{k}\right\rangle=0$ for all $m \in \Delta$. Therefore $\sum_{k=1}^{n+1} c_{k} v_{k}=0$. this combined with $\sum c_{k}=0$ implies that $\sum_{k=1}^{n+1} c_{k} \tilde{v_{k}}=0$ which contradicts the linear independence of $\tilde{v_{1}}, . ., v_{n+1}$. Thus the lemma holds.

So we have the following theorem.

Theorem 4 For any generic nondegenerate anticanonical hypersurface $V$ of an $n$-dimensional simplicial Fano toric variety $\mathbb{P}_{\Delta}$, and $n \geq 4$,

$$
h^{n-2,1}(V)=l(\Delta)-n-1-\sum_{\operatorname{dim}(F)=n-1} l^{*}(F) .
$$

Note : $F$ denotes a facet of the polytope $\Delta$ in the statement of the above theorem.

\subsection{Cohomology of the twisted sectors of $V$}

We now want to compute $h^{n-3,0}\left(V_{(g)}\right)$ for any twisted sector $V_{(g)} \cong V \cap \mathbb{P}_{\widehat{F}^{\circ}}$. This is obviously zero if $\operatorname{dim}\left(F^{\circ}\right)>1$, since $\operatorname{dim}\left(\mathbb{P}_{\widehat{F^{\circ}}}\right)=n-1-\operatorname{dim}\left(F^{\circ}\right)$. So we will only consider the case $\operatorname{dim}\left(F^{\circ}\right)=1$. Let $\tau$ be the 2-dimensional cone obtained by coning over $F^{\circ}$. As noted earlier $\bar{O}_{\tau}=\mathbb{P}_{\widehat{F^{\circ}}}$. The restriction of $V$ to $\overline{O_{\tau}}$ gives a quasismooth ample hyprsurface of $\overline{O_{\tau}}$, which we shall identify with $V_{(g)}$. So we are again in a situation where we can invoke lemma 2.

For this we need to understand the homogeneous coordinate ring $S^{\prime}$ of $\overline{O_{\tau}}$. According to Fulton[[8], section 3.1], a fan for $\overline{O_{\tau}}$ can be constucted from the fan $\Xi$ of $X$ as follows.

Let $N_{\tau}$ be the sublattice of $N$ generated by the primitive one dimensional generators of $\tau$. Let $N(\tau)=N / N_{\tau}$. The dual lattice of $N(\tau)$ is given by $M(\tau)=\tau^{\perp} \cap M$. The star of a cone $\tau$ can be defined abstractly as the set of cones $\sigma$ in $\Xi$ that contain $\tau$ as a face. Such cones $\sigma$ are determined by their images in $N(\tau)$ i.e. by $\bar{\sigma}=\left(\sigma+\left(N_{\tau}\right)_{\mathbb{R}}\right) /\left(N_{\tau}\right)_{\mathbb{R}} \subset N_{\mathbb{R}} /\left(N_{\tau}\right)_{\mathbb{R}}=N(\tau)_{\mathbb{R}}$. These cones $\{\bar{\sigma}: \tau<\sigma\}$ form a fan $\operatorname{Star}(\tau)$ in $N(\tau)$. $\bar{O}_{\tau}$ is the toric variety corresponding to this fan. 
Wlog let $v_{1}, v_{2}$ be the generators of $\tau$. The corresponding Weil divisors in $X$ are $D_{1}$ and $D_{2}$, and $\bar{O}_{\tau}$ is the intersection $\left|G_{\tau}\right| D_{1} D_{2}$ considered as an element of the Chow ring of $X$.

Again wlog assume that $v_{j}, j=3, . ., l$ are the one dimensional cones of $\Xi$ such that $\left\{v_{1}, v_{2}, v_{j}\right\}$ generate a three dimensional cone of $\Xi$. In other words, $\bar{v}_{j}, j=3, . ., l$ are the one dimensional cones of $\operatorname{Star}(\tau)$. Let $\tilde{D}_{j}:=D_{1} D_{2} D_{j}$ for $j=3, . ., r$. Note that $\tilde{D}_{j}=0$ if $j>l$. The divisor of $\overline{O_{\tau}}$ corresponding to $\bar{v}_{j}$ is $\tilde{D}_{j}$ for $j=3, \ldots, l$. So the homogeneous coordinate ring $S^{\prime}$ is generated by variables $y_{j}$ corresponding to $\tilde{D}_{j}$ for $j=3, . ., l$. Denote by $\alpha_{0}$ the anticanonical class $\sum_{j=3}^{l} \tilde{D}_{j}$ in $S^{\prime}$.

On the other hand the divisor $V$ restricts to $-K_{X} D_{1} D_{2}=\left(D_{1}+\ldots+\right.$ $\left.D_{r}\right) D_{1} D_{2}=\sum_{j=3}^{l} \tilde{D}_{j}+\left(D_{1}+D_{2}\right) D_{1} D_{2}$. To see what $\left(D_{1}+D_{2}\right) D_{1} D_{2}$ is in terms of the $\tilde{D}_{j} \mathrm{~s}$, we can pick a point $m \in \widehat{F^{\circ}} \cap M$ and let $b_{i}:=\left\langle m, v_{i}\right\rangle, 1 \leq$ $i \leq r$. Then $\sum_{i=1}^{r} b_{i} D_{i}$ is linearly equivalent to zero. Note that $b_{1}=b_{2}=-1$. Hence we have $D_{1}+D_{2}=\sum_{i=3}^{r} b_{i} D_{i}$. So, $\left(D_{1}+D_{2}\right) D_{1} D_{2}=\sum_{i=3}^{l} b_{i} \tilde{D}_{i}$. Let $\alpha$ be the class in $S^{\prime}$ representing the effective ample divisor $-K_{X} D_{1} D_{2}$. Let $f^{\prime}$ be the associated homogeneous polynomial in the $y_{j}$ s. Now we can apply lemma 2 to the $(n-2)$-dimensional variety $\bar{O}_{\tau}$ and the ample hypersurface $V_{(g)}$. Choose $k=1$ in the lemma to get

$$
\left(S^{\prime} / J\left(f^{\prime}\right)\right)_{\alpha-\alpha_{0}} \simeq P H^{n-3,0}\left(V_{(g)}\right)=H^{n-3,0}\left(V_{(g)}\right)
$$

since $H^{n-3,0}\left(\overline{O_{\tau}}\right)=0$.

Now $\alpha-\alpha_{0}=\left[\alpha-\sum_{j=3}^{l} \tilde{D}_{j}\right]$. A typical generator $\partial f^{\prime} / \partial y_{i} \in J\left(f^{\prime}\right)$ has degree $\left[\alpha-\tilde{D}_{i}\right]$. There are no nonconstant regular functions on the projective variety $\tilde{O}_{\tau}$. So any nontrivial effective divisor, and in particular $\tilde{D}_{i}, \sum_{j=3, . . l ; j \neq i} \tilde{D}_{j}$ and $\sum_{j=3, . . l} \tilde{D}_{j}$ are not linearly equivalent to zero. This implies that $\left(J\left(f^{\prime}\right)\right)_{\alpha-\alpha_{0}}=0$. Hence we obtain that

$$
\left(S^{\prime}\right)_{\alpha-\alpha_{0}} \simeq H^{n-3,0}\left(V_{(g)}\right)
$$

Now $\alpha-\alpha_{0}=\left[\sum_{j=3}^{l} b_{j} \tilde{D}_{j}\right]$. We want to identify the effective divisors in this class. So we want $m_{*} \in M(\tau)$ such that $\sum_{j=3}^{l}\left(b_{j}+\left\langle m_{*}, \bar{v}_{j}\right\rangle\right) \tilde{D}_{j}$ is effective. This is if and only if $\left(b_{j}+\left\langle m_{*}, \bar{v}_{j}\right\rangle\right) \geq 0$ for all $j=3, . ., l$

$\Longleftrightarrow\left(\left\langle m+m_{*}, v_{j}\right\rangle\right) \geq 0$ for $j=3, . ., l$

$\Longleftrightarrow m+m_{*} \in \operatorname{Int} \widehat{F^{\circ}} \cap M$. 
To justify the last step note that $\left\langle m+m_{*}, v_{i}\right\rangle=-1$ for $i=1,2$ so that $m+m_{*} \in \widehat{F^{\circ}} \cap M$. If $m+m_{*}$ is not in the interoir of $\widehat{F^{\circ}}$ then it belongs to some face $\widehat{E^{\circ}}$ where $F^{\circ} \subset E^{\circ}$ as faces of $\Delta^{\circ}$. The cone $\delta$ associated to $E^{\circ}$ contains $\tau$ as a face and hence contains some $v_{k}$ for $3 \leq k \leq l$. Then $\left\langle m+m_{*}, v_{k}\right\rangle=-1$ which is a contradiction.

Since $m$ is fixed, the required effective divisors are in one-to-one correspondence with the interior lattice points of $\widehat{F^{\circ}}$. Hence $h^{n-3,0}\left(V_{(g)}\right)=l^{*}\left(\widehat{F^{\circ}}\right)$. Since there are $l^{*}\left(F^{\circ}\right)$ twisted sectors isomorphic to $V \cap \mathbb{P}_{F^{\circ}}$ we have the following.

$h_{\text {orb }}^{n-2,1}(V)$

$=h^{n-2,1}(V)+\sum_{\iota(g)=1} h^{n-3,0}\left(V_{(g)}\right)$

$=l(\Delta)-n-1-\sum_{\operatorname{dim}(F)=n-1} l^{*}(F)+\sum_{\operatorname{dim}\left(F^{\circ}\right)=1} l^{*}\left(F^{\circ}\right) l^{*}\left(\widehat{F^{\circ}}\right)$

$=l(\Delta)-n-1-\sum_{\operatorname{dim}(F)=n-1} l^{*}(F)+\sum_{\operatorname{dim}(F)=n-2} l^{*}(F) l^{*}(\widehat{F})$.

For the last step we used the one-to-one correspondence between faces of $\Delta$ and $\Delta^{\circ}$.

\subsection{Main Results}

Combining the above formula with theorem 4 we have the following theorem.

Theorem 5 For any generic nondegenerate anticanonical hypersurface $V$ of an $n$-dimensional simplicial Fano toric variety $\mathbb{P}_{\Delta}, n \geq 4$,

$$
h_{\text {orb }}^{n-2,1}(V)=l(\Delta)-n-1-\sum_{\operatorname{dim}(F)=n-1} l^{*}(F)+\sum_{\operatorname{dim}(F)=n-2} l^{*}(F) l^{*}(\widehat{F}) .
$$

Corollary 1 If $\widehat{V}$ is an MPCP desingularisation of any generic nondegenerate anticanonical hypersurface $V$ of an n-dimensional simplicial Fano toric variety $\mathbb{P}_{\Delta}, n \geq 4$, then $h_{\text {orb }}^{p, 1}(V)=h^{p, 1}(\widehat{V})$ for $p=1$ and $p=n-2$

Proof The formulas for $h^{p, 1}(\widehat{V})$ for $p=1, n-2$ computed in [1] by Batyrev match the orbifold Hodge numbers for $V$ obtained in theorem 3 and theorem 5 .

Corollary 2 In the case $n=4, h_{\text {orb }}^{p, q}(V)=h^{p, q}(\widehat{V})$ for any $p$ and $q$. 
Proof We need only consider $p, q \leq 3$. Also $h_{o r b}^{p, 0} \equiv h^{p, 0}$ by definition since $\iota$ is nonnegative. So, by Serre duality, it is enough to consider just the cases $p=1, q=1$ and $p=2, q=1$. These are addressed by corollary 1 .

We should remark here that in this case $\widehat{V}$ is actually smooth.

Corollary 3 If $\mathbb{P}_{\Delta^{\circ}}$ is also simplicial, and $V^{\circ}$ is a generic nondegenerate anticanonical hypersurface of $\mathbb{P}_{\Delta^{\circ}}$, then $h_{\text {orb }}^{1,1}(V)=h_{\text {orb }}^{n-2,1}\left(V^{\circ}\right)$ and vice versa.

Proof Follows from interchanging the roles of $\Delta$ and $\Delta^{\circ}$ in the formulas.

Remark In particular, for the $n=4$ case, we have $h_{\text {orb }}^{p, q}(V)=h_{\text {orb }}^{3-p, q}\left(V^{\circ}\right)$. This is an example of 'mirror symmetry' of orbifold hodge numbers.

\subsection{An Example}

Consider the complex 4-dimensional weighted projective space $X=\mathbb{P}(1,1,2,2,2)$. It is a simplicial Fano toric variety. It's fan $\Xi$ has the following 1-dimensional cones in $N \cong \mathbb{Z}^{4}: v_{1}=(-1,-2,-2,-2), v_{2}=(1,0,0,0), v_{3}=(0,1,0,0)$, $v_{4}=(0,0,1,0), v_{5}=(0,0,0,1) . \Xi$ has five 4-dimensional cones, obtained by dropping one of the $v_{i}$ 's at a time and taking the cone generated by the remaining four.

Let $D_{i}$ denote the torus-invariant divisor given by the orbit closure $\overline{O_{v_{i}}}$. It is easy to check that in $A_{3}(X),\left[D_{2}\right]=\left[D_{1}\right]$ and $\left[D_{i}\right]=2\left[D_{1}\right]$ for $i \geq 3$. Construct the homogeneous coordinate ring of $X$ by introducing variables $x_{i}$ corresponding to $v_{i}$. Then $\operatorname{deg}\left(x_{1}\right)=\operatorname{deg}\left(x_{2}\right)=1\left(=\left[D_{1}\right]\right)$ and $\operatorname{deg}\left(x_{i}\right)=2$ for $i \geq 3$.

This leads to the more familiar description of $\mathbb{P}(1,1,2,2,2)$ as $\left(\mathbb{C}^{5}-\{0\}\right) / \mathbb{C}^{*}$. The action of any $\alpha \in \mathbb{C}^{*}$ on $\mathbb{C}^{5}-\{0\}$ is as follows :

$\alpha .\left[x_{1}: x_{2}: x_{3}: x_{4}: x_{5}\right]=\left[\alpha x_{1}: \alpha x_{2}: \alpha^{2} x_{3}: \alpha^{2} x_{4}: \alpha^{2} x_{5}\right]$.

In this description, $D_{i}$ corresponds to the hyperplane $\left\{x_{i}=0\right\}$ and the 4dimensional cones of $\Xi$ correspond to the open sets $\left\{x_{i} \neq 0\right\}$. It is also easily seen that the singular locus of $X$ is precisely the surface $\left\{x_{1}=x_{2}=0\right\}$. In fact, this represents the only twisted sector of $X$. The $v_{i}$ 's are the vertices of a reflexive polytope $\Delta^{\circ}$. The faces of $\Delta^{\circ}$ have only one interior lattice point : $(0,-1,-1,-1)=1 / 2\left(v_{1}+v_{2}\right)$. This lattice point corresponds to the twisted 
sector and the local isotropy group is $\mathbb{Z}_{2}$.

The dual reflexive polytope $\Delta$ in $M_{\mathbb{R}}$ has the following vertices: $w_{1}=(-1,-1,-1,-1), w_{2}=(7,-1,-1,-1), w_{3}=(-1,3,-1,-1)$, $w_{4}=(-1,-1,3,-1), w_{5}=(-1,-1,-1,3)$.

$\Delta$ is the polytope corresponding to the anticanonical divisor $-K_{X}=$ $\sum_{i=1}^{5} D_{i}$ of $X$, and $X=\mathbb{P}_{\Delta}$. If $V$ is a generic nondegenerate Calabi-Yau (anticanonical) hypersurface of $X$, then $V$ has just one twisted sector namely $V \cap\left\{x_{1}=x_{2}=0\right\}$. One can directly compute the genus of this curve by using the Riemann-Hurwitz formula. It turns out to be 3 .

The following is a list of the Hodge numbers of $V: h^{1,0}=h^{2,0}=0$, $h^{3,0}=1, h^{1,1}=1, h^{2,1}=83, h_{\text {orb }}^{1,1}=2, h_{\text {orb }}^{2,1}=86$.

The dual Fano veriety $\mathbb{P}_{\Delta^{\circ}}$ is also simplicial. This is easily checked since its fan is is obtained by coning over the faces of $\Delta$. In fact, $\mathbb{P}_{\Delta^{\circ}}=\mathbb{P}_{\Delta} / \mathbb{Z}_{4}^{3}$. This is also shown easily. First, observe that $w_{1}=-w_{2}-2 w_{3}-2 w_{4}-2 w_{5}$. Secondly, if $\bar{M}$ is the sublattice of $M$ generated by $w_{2}, w_{3}, w_{4}, w_{5}$, then $M / \bar{M}$ $=\mathbb{Z}_{4}^{3}$.

The mirror Calabi-Yau family is also easy to write down. Let $z_{i}$ denote the homogeneous coordinate of $\mathbb{P}_{\Delta^{\circ}}$ correspoding to $w_{i}$. Then a generic Calabi-Yau hypersurface $V^{\circ}$ of $\mathbb{P}_{\Delta^{\circ}}$ has equation:

$$
\lambda_{1} z_{1}^{8}+\lambda_{2} z_{2}^{8}+\lambda_{3} z_{3}^{4}+\lambda_{4} z_{4}^{4}+\lambda_{5} z_{5}^{4}+\lambda_{6} z_{1}^{4} z_{2}^{4}+\lambda_{0} z_{1} z_{2} z_{3} z_{4} z_{5}=0 .
$$

Note that $h_{\text {orb }}^{1,1}\left(V^{\circ}\right)=86$ and $h_{\text {orb }}^{2,1}\left(V^{\circ}\right)=2$.

\section{Bibliography}

[1] V.Batyrev, Dual polyhedra and Mirror symmetry for Calabi-Yau hypersurfaces in toric varieties, AG/9310003

[2] V.Batyrev, Non-Archimedean Integrals and Stringy Euler numbers of logterminal pairs, JEMS 1, 1999.

[3] V.Batyrev and D.Cox, On the Hodge structure of projective hypersurfaces in toric varieties, AG/9306011 
[4] V.Batyrev and D.Dais, Strong McKay correspondence, string-theoretic Hodge numbers and mirror symmetry, AG/9410001

[5] W.Chen and Y.Ruan, A New cohomology theory for Orbifold, AG/0004129

[6] D.Cox and S.Katz, Mirror Symmetry and Algebraic Geometry, Mathematical Surveys and Monographs, Vol 68, AMS.

[7] J.Denef and F.Loeser, Germs of arcs on singular algebraic varieties and motivic integration, Invent. Math. 135 (1999).

[8] W.Fulton, Introduction to toric varieties, Princeton University Press, 1993.

[9] M.Reid, La correspondance de McKay, AG/9911165

[10] Y.Ruan, Stringy geometry and Topology of Orbifolds, AG/0011149 